\title{
ON THE UNIFICATION OF RADIO GALAXIES AND QUASARS
}

\author{
KRZYSZTOF T. CHYZY \\ Astronomocal Observatory of the Jagiellonian University \\ ul. Orla 171, 30-244 Krakow, Poland
}

A comparison of the projected linear size evolution of extended quasars and radio galaxies are often used as a test of the radio galaxy - quasar unification schemes. If both the mentioned categories of radio sources differ to an observer only due to the various viewing directions, then their radio linear sizes are expected to evolve with redshift in the same way (Gopal-Krishna \& Kulkarni, 1992). However, apart from the simplest linear size parameter $L$ we can still determine two independent parameters assessing the asymmetry of the radio structure: $Q$ the arm lengths ratio; $M$ - misalignment, which measures the apparent bending, and defined as the ratio of the displacement of the core from the source axis to the linear size. The asymmetry parameters $Q$ and $M$ can also be a powerful tool in the consistency test for the orientation based unification scheme as their evolutionary patterns should be the same for radio galaxies and quasars. Contrary to the linear size, they are not sensitive to the simple homological rescaling of the whole structure and hence to the age or expansion velocity of the structure.

In order to derive the evolutionary behavior of the asymmetry of radio structures the dependence of the median values of $Q$ and $M$ parameter on redshift and radio luminosity has been estimated in the form $\propto(1+z)^{n}$ and $\propto P^{\beta}$ respectively for 152 powerful radio galaxies and 173 steep-spectrum quasars (Chyzy \& Zieba, 1993). The striking result of the comparison of the fitted parameters is the stronger evolution of the asymmetry $Q$ and $M$ for radio galaxies than for quasars in concordance with the faster decrease of radio galaxy linear sizes. Moreover, linear size and bending are more closely correlated with radio luminosity for radio galaxies than for quasars.

The possible causes underlying for the evolution of geometrical parameters seem to be similar for radio galaxies and quasars. For both the types of objects with increasing redshift there is a fast decrease in their sizes, increase in bending and slower increase in arm asymmetry. However, this epoch dependency is far stronger for radio galaxies than for quasars, which resembles an analogous tendency in correlation with luminosity. As radio morfology is closely connected with the state of galactic surroundings we may conjecture that the evolutionary state of galactic environment associated with quasars and radio galaxies slightly differs. A part of this effect may be caused by somewhat distinct redshift range for these objects.

\section{References}

Chyzy, K.T., Zieba, S., 1993, Astron. Astrophys.267, L27.

Gopal-Krishna, Kulkarni, V.K., 1992, Astron. Astrophys.257, 11.

501

T. J.-L. Courvoisier and A. Blecha: Multi-Wavelength Continuum Emission of AGN, 501.

(C) 1994 IAU. Printed in the Netherlands. 